\title{
ANÁlISIS TEXTUAL-DISCURSIVO Y PEDAGÓGICO-DISCURSIVO DE LA INTERACCIÓN COMUNICATIVA EN FOROS FORMATIVOS ONLINE: SOBRE LA ARTICULACIÓN LOCAL DE LAS INTERVENCIONES
}

\author{
(ANALYSIS OF COMMUNICATIVE INTERACTION OF ONLINE EDUCATION FORUM \\ FROM TEXTUAL DISCURSIVE AND DIDACTIC DISCURSIVE PERSPECTIVES: THE LOCAL \\ INTERVENTION ARTICULATION)
}

Lourdes Morán

Guadalupe Álvarez

Consejo Nacional de Investigaciones Científicas y Técnicas, CIAFIC-CONICET (Argentina)

\section{RESUMEN}

A partir de la década de 1990, el e-learning pasa de modelos de aprendizaje basados en la transmisión de conocimiento a modelos basados en la construcción de conocimiento. Este cambio se ha visto favorecido por la irrupción de las tecnologías de la información y la comunicación, que proporcionan un buen soporte para que interactúen estudiantes y tutores. Sin embargo, no cualquier modalidad de uso asegura dicha construcción. En este sentido, un aspecto fundamental a considerar está dado por las modalidades en que se desarrolla la interacción comunicativa en los foros. En relación con ello, el objetivo de este artículo es realizar un análisis de la articulación que se produce entre las intervenciones realizadas por los participantes buscando identificar si la intervención de apertura de los foros funciona como guía o patrón de las emisiones subsiguientes. El análisis muestra que existe mayor articulación entre cada emisión y las que se suceden en la secuencia, que entre ellas y la intervención de apertura.

Palabras clave: tecnologías de la información y la comunicación, interacción en línea, aprendizaje mediado por la computadora.

\begin{abstract}
Since the 1990s, e-learning has transformed from learning models that are based on the knowledge transfer into models based on knowledge creation. This change has been facilitated by the rapid introduction of new Information and Communication Technologies (ICTs). ICTs provide a solid infrastructure for students and their tutors to interact with each another. However, not all modalities
\end{abstract}


(or modes of study) can provide this type of infrastructure. One of the key elements we need to take into consideration is provided by virtual modalities, i.e. where communicative interaction is developed in online fora. The objective of this article is therefore to analyze the sequencing of online activities/tasks that are carried out by participants and to identify whether the first online task can serve as a "guide" or a "theoretical model" of subsequent online activities. Our findings show that the sequencing of online tasks is more integrated and cohesive when done consecutively than when merely based on the initial online entry.

Key words: Information and Communication Technologies (ICTs), online interaction, ITbased learning.

A partir de la década de 1990, el e-learning pasa de modelos de aprendizaje basados en la transmisión de conocimiento a modelos basados en la construcción de conocimiento, a partir de los cuales los estudiantes participan activamente en el aprendizaje y los profesores intervienen como facilitadores en la construcción y la apropiación del conocimiento (Banzato, 2002). Este cambio se ha visto favorecido por la irrupción de las tecnologías de la información y la comunicación (TIC), que proporcionan un buen soporte para que interactúen estudiantes y tutores. Sin embargo, no cualquier modalidad de uso asegura dicha construcción. En este sentido, un aspecto fundamental a considerar está dado por las modalidades en que se desarrolla la interacción comunicativa en los foros. En relación con ello, y teniendo en cuenta que la emisión iniciadora suele funcionar como guía o patrón de las emisiones subsiguientes (Pardo, 1994), hemos desarrollado un artículo anterior en que elaboramos una caracterización de la intervención de apertura de los foros desde dos niveles: un nivel discursivo multimedial y un nivel pedagógico. A partir de dicho trabajo surgió una segunda etapa de investigación, que presentamos en este trabajo. En esta etapa, partimos de la misma hipótesis considerada en la primera parte (i.e. la emisión iniciadora es guía del resto) y analizamos en qué medida y de qué maneras se articulan las intervenciones de la interacción comunicativa de los foros.

\section{SOBRE EL DISCURSO DIDÁCTICO ONLINE}

\section{La naturaleza didáctica de los foros}

Los foros de discusión o conferencias asincrónicas son una de las herramientas más utilizadas en la estructura didáctica de la formación online. En estudios anteriores (e.g. Constantino, 2002a), se han reconocido diversas variantes de uso de los foros: 
- Como forma de presentación personal individual en un espacio público común.

- Como "pizarra" o "cartelera", que permite cumplir con determinadas actividades propuestas por el tutor/profesor.

- Como espacios de trabajo para el aprendizaje, es decir, foros con finalidad didáctica en sí.

Este tipo depende de la interacción de los participantes, por lo cual es fundamental considerar el modo en que se desarrolla la interacción comunicativa entre el experto/ docente/tutor y los/las alumnos/as. Un aspecto importante, en este sentido, está representado por las intencionalidades pedagógicas.

\section{La intencionalidad pedagógica en las intervenciones de docentes y tutores}

Cuando un docente o tutor planifica las intervenciones que realizará para iniciar las actividades, plantea en primer lugar una clase de intervención que es coherente con una estrategia didáctica que busca desplegar. Esta estrategia didáctica es el reflejo de una intencionalidad didáctica que está en estrecha relación con los propósitos más específicos de la actividad. No podemos decir que existe una estrategia que sea la "correcta" para toda la enseñanza. Los docentes cuentan con una variedad de estrategias, a los fines de poder seleccionar la más adecuada, e incluso ir adecuándola a las diferentes situaciones institucionales, grupos, contenidos, etc.

Los modelos de enseñanza sistematizados por Joyce, Weil y Calhoun (2000), con los aportes de Eggen y Kauchak (2001), y los diseños instruccionales compilados por Reigeluth (1999, 2009), muestran cómo diferentes intencionalidades pedagógicas se corresponden con ciertas estrategias docentes y particulares maneras de intervenir en el desarrollo de las actividades con los alumnos.

En la formación online las intervenciones docentes pueden analizarse desde diferentes ángulos: los recursos que se utilizan, las herramientas que se activan en la plataforma, las actividades propuestas y el desarrollo de los foros. De todos ellos, el trabajo en los foros es el espacio que nos aporta información más específica sobre las estrategias didácticas concretas que llevan a cabo los docentes. En estos espacios se plantean preguntas, se presentan casos para el análisis, se invita a la reflexión, se expone información nueva, etc. Y todas estas acciones dan cuenta de intencionalidades pedagógicas diferentes. 


\section{Sobre el análisis del discurso en los foros formativos online}

Una decisión importante para quien se enfrenta al estudio de foros es la elección de la metodología y el modelo para el análisis del discurso presente en ellos. Como han señalado y estudiado Gros Salvat y Silva (2006), existe una amplia variedad de perspectivas y modelos para ese estudio (Sánchez-Upegui, 2009; Garrison y Anderson, 2005; Gunawardena y otros, 1997; Mercer, 2000; Silva, 2006), y esto implica diversidad de unidades de análisis.

En cuanto a los estudiosiniciales del análisis del discurso didáctico (Davisy Brewer, 1997), se han dedicado a analizar la colaboración a partir de los datos cuantitativos de las intervenciones, sin analizar en profundidad el contenido de la interacción ni las consecuencias de las modalidades de interacción en el aprendizaje.

Recientemente, en cambio, los estudios (Constantino, 2000a, 2007; SánchezUpegui, 2009; Silva, 2006) han profundizado el estudio del contenido y la modalidad de interacción comunicativa en los foros, y han comenzado a ocuparse de sus consecuencias en el aprendizaje.

En este sentido, se han ofrecido clasificaciones para diferenciar el grado o nivel de actividad interactiva que se desarrolla en los foros. En particular, se ha desarrollado una propuesta clasificatoria de los modos o estrategias de participación diferenciales encontradas en una investigación con foros de diferente tipo (Constantino, 2002a):

- Participación aditiva o relativa al tópico del foro.

- Participación interactiva o relativa al flujo discursivo.

- Participación directiva o tendiente a mantener el flujo discursivo en el tópico.

- Participación disruptiva o relativa a tópicos diversos.

- Participación anómala o inesperada.

- Participación recapitulativa, quesintetiza oreconsideratópicosy/ocontribuciones previas, presentadas en forma articulada.

- Participación generativa o creativa, que implica la propuesta de amplificaciones temáticas, perspectivas de análisis diferentes, detección de necesidades u obstáculos conceptuales, estimación de resultados, proyección de alternativas, etc. 
Muy recientemente, Sánchez-Upegui (2009) ha planteado un marco conceptual y metodológico para el análisis de foros que está centrado en elementos del discurso especializado de carácter educativo, como el análisis lingüístico de la interacción mediatizada por computador, la ciberpragmática, la teoría de la cortesía, la argumentación y el principio cooperativo de la comunicación. A partir de este tipo de análisis, ha concluido que la formación de estudiantes y docentes en estrategias de argumentación, corrección lingüística e interacción, desde una perspectiva lingüístico-textual y ciberpragmática, es imprescindible para el desarrollo de competencias colaborativas, comunicativas y científicas en la educación superior virtual. Pero, si bien el análisis propuesto Sánchez-Upegui (2009) ilumina diferentes aspectos de la dinámica discursiva en los foros, su corpus es pequeño, por lo cual resulta necesario considerar un corpus más amplio en el cual aplicar conceptos provenientes del análisis del discurso.

Sin duda, las propuestas desarrolladas recientemente constituyen una aproximación válida al análisis de la actividad discursiva de los participantes en foros, en cuanto reflejan acciones y actitudes comunicativas y cognitivas que se concretan a través de los textos que ellos producen. No obstante, ninguna de ellas se ha dedicado especialmente a analizar el modo en que se articulan las intervenciones a lo largo de la interacción comunicativa. Esta dimensión nos ha resultado sumamente importante teniendo en cuenta que este trabajo parte de la hipótesis de Pardo (1994), a partir de la cual se postula que todo texto tiene una emisión líder, generalmente la iniciadora, que puede funcionar como guía o patrón de las emisiones subsiguientes. Si bien no hemos recurrido a las categorías con las cuales la autora caracteriza la emisión líder, sí nos ha interesado pensar en la posibilidad de que la primera intervención de un foro condicione el desarrollo de las posteriores intervenciones. Este estudio ha requerido considerar qué conceptos permiten el análisis de la interacción comunicativa desde un punto de vista tanto textual-discursivo como discursivo-pedagógico.

\section{Conceptos para analizar la interacción comunicativa desarrollada en los foros desde un punto de vista textual-discursivo}

Definir un modelo para analizar la interacción comunicativa, sea presencial u online, requiere adoptar una perspectiva dialógica, es decir, una perspectiva a partir de la cual las acciones solo se reconocen en el marco de un proceso dialógico con los contextos y los interlocutores, y en virtud de la verbalización que las hace posibles (Linell, 1998, p. 93). Desde esta perspectiva se asumen tres principios fundamentales (Linell, 1998; Marková y Linell, 1996): 
- Secuencialidad: la intervención de un hablante no puede ser comprendida si se la aísla del resto de las intervenciones del mismo hablante y de otro/s interlocutor/ es. Por el contrario, las emisiones están secuencialmente organizadas y su significado depende en parte de su posición en la secuencia (que en la interacción en tiempo real es, por naturaleza, de orden temporal), y de la articulación con los significados de las emisiones tanto precedentes como siguientes (cotexto).

- Construcción compartida: el lenguaje utilizado en la comunicación tiene un origen social, tanto por su génesis histórica como por ser distribuido, negociado y recreado en la interacción. Esta construcción colectiva es posible por las acciones e interacciones que los actores coordinan mutua y recíprocamente.

- Dependencia de actividad o coconstitución del acto-actividad: actos, emisiones o secuencias en el discurso que están esencialmente situados dentro de una actividad donde los interlocutores lo producen de manera conjunta. Esta actividad puede a menudo ser vista como un tipo general o perteneciente a un género particular ${ }^{1}$.

A partir de estos principios básicos, llamaremos intervención a cada participación de los profesores, tutores y alumnos en el foro, y analizaremos estas intervenciones en dos dimensiones. Por un lado, la dimensión accional, determinado por la/s acción/ es que propone realizar el participante y, los recursos y las estrategias ${ }^{2}$ empleados para ello. Por otro lado, la dimensión conceptual, caracterizada por las ideas y los conceptos desarrollados en cada intervención y, los recursos y las estrategias empleadas para dicho desarrollo.

\section{Algunas observaciones relativas a la dimensión accional de las intervenciones}

La dimensión accional del lenguaje ha sido estudiada por varios autores, sobre todo, en los ámbitos de la filosofía y la lingüística (Austin, 1962; Fetzer, 2002; Mey, 1993; Reiss 1985; Sbisà, 1992; Searle, 1969, 1975, 1979). Los diferentes estudios han hecho evidente que, al utilizar formas lingüísticas en una situación determinada, se pueden realizar acciones. También han mostrado que la relación que se establece entre el lenguaje y las acciones sólo se puede comprender a partir de diversos factores, tanto lingüísticos como no lingüísticos. En este sentido, se ha logrado: a) reconocer diferentes factores lingüísticos comprometidos en las acciones realizadas, como la prosodia, la entonación, la sintaxis o el léxico; b) identificar diferentes fuerzas asociadas con las acciones que, convencionalmente, pueden realizar los hablantes (afirmación, orden, pedido, sugerencia, etc.); c) establecer posibles reacciones 
que se espera que realicen los oyentes en virtud de las acciones propuestas por los hablantes.

Partiendo de estas observaciones, en esta parte del trabajo nos ocuparemos de identificar las acciones que los hablantes realizan por medio de formas lingüísticas en tanto recursos, es decir, formas que el hablante pone en funcionamiento dentro de una situación determinada y con una finalidad específica. Es importante tener en cuenta que cada acción, en lugar de ser reconocida de manera aislada, se identificará en el marco de la interacción comunicativa, es decir, considerando una serie de acciones que están secuencialmente presentes en dicha interacción.

\section{Conceptos para analizar la interacción comunicativa desarrollada en los foros en función de objetivos pedagógicos}

Considerar el desarrollo del diálogo entre docentes y alumnos en función del tratamiento de los contenidos, en el marco de un proceso de enseñanza y aprendizaje, requiere pensar en un diálogo con características pedagógicas.

Entre las características que debe asumir el diálogo para que sea pedagógico en primer lugar se cuenta la necesidad de contar con la participación activa de todos los interlocutores. La participación activa puede aceptar una variedad de formas pero, puesto que el propósito del diálogo es el trabajo conjunto con el contenido, debe haber oportunidad para el compromiso, para el cuestionamiento, para someter a prueba las nuevas ideas y para escuchar los diferentes puntos de vista. La principal amenaza a ese fin, como tan bien lo ha mostrado Freire (2000) es el monólogo, la presentación de un único y autoritario punto de vista que no admite cuestionamientos ni tolera la participación. La participación de los involucrados en una relación comunicativa de esta clase debe ser algo voluntario y abierto a la intervención activa de los participantes. Todos los interlocutores tienen que estar habilitados para introducir temas, formular preguntas, poner en discusión otros puntos de vista o iniciar cualquiera de las demás actividades que definen la relación dialógica.

Otra característica que parece central para el diálogo es, como recién mencionamos, el compromiso. Entrar en una relación comunicativa de esta clase tiene que garantizar un flujo de conversación persistente y amplia, que cruce preocupaciones comunes, aún difíciles y conflictivas. Este principio requiere, además, una entrega y confianza en el proceso comunicativo suficientes para estar dispuestos a revelar nuestros sentimientos, nuestras razones y motivaciones si se nos pregunta 
por ellos. Burbules (2000) se refiere a este aspecto como un acto de comprensión y aceptación del otro en la situación de comunicación interpersonal.

Finalmente, entre las condiciones para el desarrollo del diálogo pedagógico podemos considerar la reciprocidad: se debe entrar en una relación comunicativa de esta clase con un espíritu de respeto y de interés mutuo, y no se deben dar por sentados papeles de privilegio o de especialización. Un corolario de este principio es que toda dinámica dentro de una relación dialógica, debe ser reversible y reflexiva. Dicho de otra manera, debemos estar preparados para que los otros nos pregunten lo que les preguntamos a ellos, y a que se espere de nosotros lo que esperamos de los demás. Si hacemos preguntas a los otros, ellos pueden hacernos preguntas; si nos ofende que los otros no presten atención a lo que decimos, debemos asegurarnos de que nos hemos esforzado por escucharlos.

\section{Diferentes tipos de diálogos que pueden darse en la enseñanza}

Burbules en su texto El diálogo en la enseñanza destaca cuatro tipos de diálogos que pueden darse dentro de situaciones de intercambio entre las personas. Esta tipología puede adaptarse a diferentes situaciones de aprendizaje y articularse con las intenciones y estrategias que los docentes despliegan para el desarrollo de sus clases.

El primer tipo de diálogo presentado es el diálogo como conversación. Este se guía claramente por el espíritu de simpatía y tolerancia ante puntos de vista distintos del propio en los momentos de intercambio. Sin este principio fundamental la conversación no es posible.

Un segundo tipo de intercambio presentado es el diálogo como indagación. Este tipo de diálogo está animado por la obtención de un nuevo conocimiento, el acuerdo en temas morales o políticos, o la solución de problemas. Es un diálogo que focaliza en el proceso de descubrimiento y de búsqueda mediante procesos de razonamiento.

Otro tipo de diálogo presentado es el diálogo como debate. Este tipo de diálogo generalmente es utilizado como estrategia privilegiada para el desarrollo del pensamiento crítico. Los debates no siempre son dialógicos, pero pueden ser dialógicos cuando las respectivas posturas abogadoras se adoptan con el espíritu de avanzar hasta una comprensión mutua de las cuestiones, y no con el de "ganar" ante todo o de hacer que el interlocutor quede mal. 
Finalmente, el diálogo como enseñanza es un tipo de diálogo en el cual el propósito de las preguntas, y de otros enunciados, es hacer que la discusión avance hacia una conclusión determinada. Basado en nociones constructivistas del saber y en modelos vigotskyanos del desarrollo, este enfoque relaciona a maestro y alumno en procesos marcadamente interactivos de interrogación.

\section{El proceso de construcción de conocimiento}

Una línea de las teorías actuales del aprendizaje es la derivada de la llamada corriente socio-histórica (Perkins, 1997; Salomón, 1993, 2002; Wenger, 1998) que considera al conocimiento como un proceso de construcción social en al menos tres sentidos:

- La creación de nuevos saberes es una actividad colectiva que, tal como sucede en el proceso de construcción del conocimiento científico, no puede ser llevada a cabo por un individuo particular y aislado;

- La socialización y el desarrollo de las personas supone una aproximación a conocimientos históricamente acumulados por una sociedad;

- El aprendizaje, en cuanto construcción o reconstrucción de conocimientos, que las personas realizan para conocer el mundo se apoya en un esfuerzo conjunto y en la interacción con otros.

En este proceso de construcción cognitiva y social las intervenciones de los docentes resultan fundamentales. El docente debe contar con habilidades específicas para conducir y guiar el proceso cognitivo de los alumnos. Entre las estrategias que resultan más significativas para el desarrollo de este proceso se pueden enumerar: recuperar el conocimiento previo de los alumnos, centrar la atención en algún aspecto en particular del proceso de aprendizaje, promover el seguimiento cognitivo ayudando a los estudiantes a reflexionar acerca de sus procesos de comprensión y pensamiento, cuestionar el grado de comprensión alcanzado, debatir con otros las propias ideas. Estas estrategias se manifiestan en los intercambios que mantienen docentes y alumnos en las diferentes situaciones de enseñanza y aprendizaje que se desarrollan. 


\section{METODOLOGÍA}

En consonancia con las cuestiones planteadas y el objetivo propuesto, la metodología es de carácter netamente cualitativo (Constantino, 2002b; Guba y Lincoln, 1998; Vasilachis de Gialdino, 2007) y encuadrable en una perspectiva dialógica-discursiva (Linell, 1998; Marková y Linell, 1996), y cognitiva-discursiva (Baquero, 1997; Burbules, 2000; Mercer, 1997).

El corpus principal de la investigación proviene del Máster Interuniversitario en Formación de Profesorado de Calidad para la Docencia Preuniversitaria (MIFORCAL) ${ }^{3}$. La estructura curricular adoptada para el proyecto, corresponde a una concepción epistémica y de organización didáctica, que distingue y articula tres niveles de conocimientos diferentes y complementarios: Teoría, Didáctica y Laboratorio (identificados como T, D y L, respectivamente). Por T, se entienden los fundamentos históricos-epistemológicos de la disciplina o área disciplinar propuesta; la $\mathrm{D}$ es concebida sintéticamente como la teoría de la praxis o acción docente también contextualizada por la disciplina o área disciplinar propuesta más otros factores propios de la situación de enseñanza; la L es conceptualizado como los conocimientos aplicados a circunstancias determinadas en contextos concretos (diseños, planes o proyecciones, simulaciones, estudios de caso, etc.). Esta tríada (T-D-L) se repite en todas las secciones temáticas del Área Común y de los Ciclos de Formación Orientados con diverso peso específico.

Para este trabajo nos centraremos en una de las orientaciones del Máster: Ciencias Sociales y Humanas. Esta orientación cuenta con diferentes materias de T, D y L, y cada una de ellas se desarrolla en uno o varios módulos. En este trabajo analizamos, particularmente, los foros correspondientes a los tres Laboratorios de esta orientación: Laboratorio de las Ciencias Sociales, Laboratorio de Didáctica de las Ciencias Humanas y Laboratorio de Metodología de la Investigación en Ciencias Sociales. A lo largo del trabajo, nos referiremos a ellos como L1, L2 y L3, respectivamente.

Esta investigación se lleva a cabo por fases, durante las cuales se aplican diferentes métodos y técnicas para el relevamiento y el análisis de los datos:

- Recolección y sistematización del corpus.

- Observación y lectura analíticas y sucesivas de los Laboratorios. 
- Análisis de las intervenciones realizadas por los participantes, considerando los conceptos señalados y prestando atención a las articulaciones entre ellas.

- Identificación de los tipos de articulaciones entre intervenciones en los foros de los laboratorios.

\section{ANÁLISIS}

El análisis ha hecho evidente, por un lado, que en los tres laboratorios se desarrollan foros diferentes según el objetivo propuesto en cada uno:

- Foro para expresar expectativas sobre el laboratorio.

- Este tipo de foro se presenta fundamentalmente en L3. Se establece un diálogo entre alumno y docente, o entre alumno y tutor.

- Foro para realizar consultas sobre el módulo o sobre la actividad.

- Este tipo de foro se presenta fundamentalmente en L3. Se establece un diálogo entre alumno y docente, o entre alumno y tutor.

- Foros enfocados en las actividades de los módulos.

- Este tipo de foro se da en L2 y L3. En estos casos, lo más frecuente es que los alumnos intervengan con participaciones breves en las que aclaren algún aspecto de la actividad realizada y adjunten esa actividad a la participación.

- Foro enfocado en el contenido y los temas de los módulos.

- Este tipo de foro se da en los tres laboratorios. En estos casos, es frecuente que haya muchas participaciones de los alumnos y que aumente la interacción entre ellos y con los tutores.

- Foro sobre la evaluación del Laboratorio.

- Este tipo de foro se da en el L3. Hay mucha participación de los alumnos, aunque no establece interacción entre ellos.

Por otra parte, en cuanto al análisis de la interacción comunicativa desarrollada en los foros, hemos notado que las articulaciones más evidentes no se establecen entre la primera intervención y la interacción comunicativa subsiguiente, como se había hipotetizado inicialmente, sino que se van estableciendo diferentes "articulaciones 
a nivel local" a lo largo de todo el intercambio. Cuando hablamos de "articulación local", nos referimos a diferentes aspectos en los cuales una participación puede influir y enmarcar la construcción de otra. Esta articulación entonces afecta tanto la dimensión accional, como la conceptual. Así, cada participación puede articularse con las participaciones que se producen seguidamente en términos de las acciones, y los conceptos en los modos que exponemos a continuación.

\section{Análisis de la dimensión accional de las intervenciones}

Como hemos adelantando, al enfocar la dimensión accional de las intervenciones, hemos relevado las acciones (orden, sugerencia, pedido, afirmación, etc.) que los hablantes realizan por medio de recursos lingüísticos y, para ello, hemos tenido en cuenta cada acción en el contexto amplio en que es realizada, o sea, consideramos las acciones previas y posteriores realizadas durante las interacciones comunicativas en los foros. De acuerdo con este relevamiento, detectamos dos tipos de situaciones muy frecuentes en que una intervención está articulada con otras en una secuencia de acciones.

Por un lado, observamos un tipo de situación ampliamente mencionada y analizada en el ámbito de la lingüística y el análisis del discurso. ${ }^{4}$ En particular, se ha identificado que la acción realizada en una intervención suele condicionar lo dicho en la intervención siguiente. En otros términos, la aparición de la primera intervención requiere no sólo que aparezca la segunda sino que esta sea de un tipo particular. Por ejemplo:

\section{Ejemplo 1}

¿Por qué estudiar y enseñar Sociología?

de Vanesa Bono - lunes, 6 de abril de 2009, 16:22

iSoy Vanesa! "brasileira, minha língua mãe é o português", pero amo el español, y si a veces me faltan sinónimos para algunas palabras y la gramática me haga una "gambeta", confío en que trabajando juntos nos entenderemos perfectamente. Me he diplomado en Tecnologías y métodos de formación en red: Tutor on-line, por la Universidad de Salamanca, España en colaboración con la Università Ca' Foscari di Venezia, y estoy cursando el doctorado en la Pontificia Universidad Católica Argentina - UCA.

Es con gran alegría que estoy recorriendo este camino, con la compañía de cada uno de ustedes y de la Coordinadora Profesora Yolanda Aguilar y del Coordinador Didáctico Omar Pen, siempre muy entusiasmados. Que podamos juntos seguir con constancia 
nuestra amistad virtual - real, juntando fuerzas, y avanzando en la interacción reflexiva y aprendizaje compartido.

i!Vamos todos juntos!! ya está lanzada nuestra próxima aventura, por los zigzagueantes senderos del espacio virtual, para animarnos e invitamos a la lectura y discusión del texto Didáctica de las Ciencias sociales: por una práctica participativa de la Profesora Andrea Tei con sus conocimientos y experiencias. Somos llevados a pensar en las cuestiones como ¿Por qué estudiar Sociología?; Enseñar sociología: una propuesta educativa y una Sociología más participativa?

Seguro que es fructífero...

Buen trabajo y cariños,

Vanesa

En este caso, la tutora, además de presentarse y mencionar sus relaciones con otros responsables del Máster, alude al material del módulo y anticipa la actividad para el foro. Esta actividad es expuesta a partir de una pregunta.

A continuación los alumnos intervienen, primero, para saludar a la docente (ejemplo 2) y, luego, para responder a la pregunta planteada (ejemplo 3).

\section{Ejemplo 2}

Re: ¿Por qué estudiar y enseñar Sociología?

de Miryan Gabina Lopez de Achar - lunes, 6 de abril de 2009, 20:10

Seguro que sí participaremos profesora, un gusto en saludarla y conocerla virtualmente, será sumamente agradable trabajar la metodología de la investigación acción a ritmo de samba.

Desde Ciudad del Este, cordial saludo de

Marta

\section{Ejemplo 3}

Re: ¿Por qué estudiar y enseñar Sociología?

de Arturo Ramal - martes, 7 de abril de 2009, 02:05

Hoola Compañer@s y Prof. Vanesa

aquí presento un primer comentario introductorio sobre el tema 
¿Por qué estudiar y enseñar Sociología?

"Para proporcionar al individuo una visión crítica del mundo em que vive. Sin Duda, ese es un punto importante y necesario, aunque a los ojos del alumno de enseñanza media eso pueda parecer un tanto abstracto”.

Primeramente debo decir que esta respuesta que aparece en el texto me sorprende en el sentido de que me parace -y esto es desde mi visión filosófica- más bien una respuesta filosófica que sociológica. Ello se debe a que la expresión "proporcionar una visión crítica del mundo en que vive" representa para mí una universalidad del saber humano. Y la sociología es una ciencia -desde mi visión y de muchos pensadores- particular que respondería a las relaciones del hombre en su entorno. El mundo en que vive el hombre no es sólo sociológico, pues también su mundo es un complejo de elementos o situaciones que condiciona su vivir. Por ejemplo la visiçon religiosa, la trascendencia, lo metafísico, lo histórico, lo existente, etc. De allí, me parece -respetando los principios comtiano y las ideas del autor de este texto- que esta respuesta es muy compleja.

Pero entrando en la propuesta de la pregunta, estudiar sociología resulta muy relevante en el sentido e que desde su postura gnoselógica se puede enseñar y comprender los aspectos estructurales de la sociedad en que vive el hombre. Y esto me parece esencial pues el hombre debe conocer su medio y las estructuras que condicionas sus relacionamientos como las leyes, las normas los aspectos culturales, etc. Es evidente que la sociología es una ciencia que puede responder a los cuestionamientos humanos que están dentro de lo social

Saludos.

Además de pregunta-respuesta o saludo-saludo, este tipo de articulaciones locales pueden ser establecer diferentes tipos de relaciones entre las intervenciones: orden-acatamiento, pedido-cumplimiento de lo solicitado, etc.

Junto con este tipo de articulación, hemos identificado otro tipo de articulación de las intervenciones a nivel accional. En particular, notamos que un participante, con frecuencia alumno, realiza una acción y, a continuación, otros realizan el mismo tipo de acción.

Para ejemplificar este caso, nos referimos al inicio de uno de los foros en el cual la tutora propone una actividad (cada subgrupo formado por la tutora "ha de desarrollar el pensamiento de un filósofo visto desde el modelo de racionalidad que manifiesta”) y los estudiantes realizan consultas puntuales sobre esa actividad antes de cumplir lo solicitado. En el marco de esta serie de intervenciones, participa un alumno con la siguiente intervención: 


\section{Ejemplo 4}

Re: Segunda semana de Laboratorio-Hegel, un modelo de racionalidad de Juan José Pena - sábado, 13 de diciembre de 2008, 12:51

Profesora, en cuanto al modelo de racionalidad hegeliana la propuesta de Grasso es bastante interesante, pero aplicable sólo a los conocidos como "grandes filósofos", aquellos que han desarrollado sistemas filosóficos, y que están descritos en los modelos citados por Grasso (el de racionalidad especulativo, el de racionalidad científico, el de racionalidad ontológico y el de racionalidad crítico).

Pienso nada mas en un filósofo como Wittgestein, cuyo sistema de pensamiento es dividido en partes, al punto que se reconoce a un Wittgestein 1 y a un Wittgestein 2, necesitaríamos dos modelos de racionalidad para explicar su filosofía?

Aunque la descripción de los modelos de racionalidad permiten una profundización en los sistemas filosóficos, no me queda claro como puede ser aplicado a las ciencias humanas. Podría darnos algunas luces al respecto?

Gracias.

En esta intervención, el alumno, en lugar de consultar una duda acerca de cómo realizar la actividad, interviene cuestionando algunos principios que subyacen a la misma. En particular, plantea la dificultad de aplicar el modelo de racionalidad propuesto por el docente de la materia a filósofos que no pertenezcan a la categoría de "grandes filósofos".

Después de que la profesora responde a la intervención de este estudiante, participa una alumna de la siguiente manera:

\section{Ejemplo 5}

Re: Segunda semana de Laboratorio-Hegel, un modelo de racionalidad de Ana Torres - lunes, 15 de diciembre de 2008, 03:46

Hola profesora y compañeros!

Estoy tratando de ponerme al día con la lectura de los foros para mañana seguir con las tareas luego de un fin de semana en que tuve que dedicarme a mis quehaceres hogareños y a lo eventos familiares de esos a los que no se puede no asistir (cumple y Bautismo!). 
Leyendo las participaciones "forísticas" me puse a pensar en este módulo de "Didáctica de las ciencias sociales" y en las actividades planteadas. Espero no lo tomen a mal el comentario que sigue... pero por lo que recuerdo somos sólo 4 filósofos en todo el grupo. Y la actividad que se plantea implica conocimientos en filosofía y sobre todo ser docente de estas asignaturas (porqué sino de qué sirve practicar didáctica en temas filosóficos?) por lo cual sólo es útil y realizable por los que nos especializamos en ella.

Digo.. las ciencias humanas no son sólo la filosofía, por lo cual los demás compañeros podrían intentar aplicar la metodología propuesta por el Prof. Grasso a autores de sus especialidades y no a filósofos que seguramente no conocen en profundidad...estamos hablando de un Wittgenstein!, un Kant!, un Aristóteles!.. etc. que yo que estudié 5 años filosofía (y sólo filosofía) no domino lo suficiente.. no quiero creer la dificultad para alguien que estudió Cs. de la Educación! Es como que a mi me pongan a hacer este trabajo pero sobre Vigotsky! (y eso que aprendí bastante de él en este curso.. pero lo mismo no lo domino..).

O apuntamos a tratar muy por arriba a los filósofos (lo cual me da idea de cierta desprolijidad profesional) o nuestros compañeros están haciendo un curso acelerado de filosofía, lo cual no creo que sea la idea de este módulo..

Sinceramente, no veo la utilidad de que todos hagamos una clase de filosofía cuando sólo 2 enseñamos filosofía.. sería más útil que cada uno practique cómo preparar una clase mejor en lo que cada uno enseña y en la disciplina que domina..

Un beso grande a todos y ojalá no tomen a mal mi comentario.

Ana

Como vemos, al igual que en la intervención previa del estudiante, la alumna tampoco consulta una duda sobre la actividad por la tutora, sino que cuestiona algunos principios que rigen dicha tarea. En este caso, cuestiona la naturaleza de la actividad (reflexión sobre filosofía) teniendo en cuenta la formación de los estudiantes (solo dos son filósofos).

Este ejemplo muestra de qué manera un participante puede "copiar" ciertos aspectos, como los accionales, de la intervención de un compañero. Por otra parte, el ejemplo hace evidente una característica de este tipo de fenómeno: "la copia" es frecuente cuando las acciones realizadas ponen en juego la imagen social (Brown y Levinson, 1987) $)^{5}$ de los participantes o de algunos de ellos. Es decir, los participantes tienden a realizar actos que amenazan la imagen social de otros, es decir, actos que pueden generar algún conflicto en el foro, cuando un compañero ha realizado previamente un acto de este tipo. 
Este fenómeno, al que podemos llamar "articulación en espejo" no solo se hace evidente en la dimensión accional sino también, como veremos en el próximo apartado, en la dimensión conceptual.

\section{Análisis de la dimensión conceptual de las intervenciones}

En cuanto al trabajo con el contenido conceptual, las primeras intervenciones de los participantes se dirigen al trabajo concreto con el contenido propuesto, en función de responder a los pedidos, a las preguntas o actividades propuestas por los tutores. En las siguientes intervenciones se observa la inclusión de temas nuevos (vinculados con el inicial propuesto), y reflexiones personales de los participantes que pasan a formar parte de las discusiones y debates. Finalmente, las intervenciones que aparecen cerca del cierre de los foros tienden a ser una conclusión o resumen de lo trabajado, ya sea motivado por los tutores o por los alumnos.

Entre las intervenciones que se fueron identificando, la articulación que se observó con mayor frecuencia fue la que articulaba la intervención de un participante que se refiere al contenido propuesto por el tutor, y las intervenciones de otros que consideran esta idea, la analizan, la retoman, la cuestionan.

En el siguiente ejemplo (ejemplo 6) se observa la intervención de una participante y a continuación dos intervenciones más (ejemplos 7 y 8) que comentan lo señalado por la primera.

\section{Ejemplo 6}

Re: Presentación del Laboratorio de Didáctica de las Ciencias Humanas de María - jueves, 4 de diciembre de 2008, o0:02

Hola todos y Prof.: Estoy leyendo el material “Ejercitaciones para filosofar, me resulta interesantísimo porque como dice el texto, es para ayudar al docente a repensar, cuales metodologías podrían realmente llevar al estudiante a "hacer filosofía", oponiéndose al puro nivel del mecanicismo, sino que lo estimula a producir, a moverse, a actuar, motivándolo a la reflexión sobre los conocimientos.

Bien dicho está, que para hacer esto, es necesario prepararse para hacer trabajar a los estudiantes y las lecciones catedráticas no se reduzcan a un estado de pasividad.

En el mismo objetivo del trabajo, sugiere partir de un hecho particular, concreto y contextualizado, de una situación histórica bien definida. 
Estas recomendaciones siempre las tenemos, pero al llevar al aula es donde pierde su practicidad.

Saludos. María.

\section{Ejemplo 7}

Re: Presentación del Laboratorio de Didáctica de las Ciencias Humanas

de Juana - jueves, 4 de diciembre de 2008, 02:53

Interesante presentación María, me ayuda a pensar los textos. Como vos decís plantea un gran desafío para el docente, repensar sus prácticas desde las prácticas metodológicas. Sabemos que no es fácil, pero el texto justamente plantea el motivar la reflexión de los alumnos y creo que por ese camino también a la propia.

Fuerza a todos.

\section{Ejemplo 8}

Re: Presentación del Laboratorio de Didáctica de las Ciencias Humanas

De Ana- jueves, 4 de diciembre de 2008, 06:45

Hola a todos!

Coincido con lo que expusieron María y Juana en cuanto a la propuesta del texto que estamos trabajando, esta manera de ayudar al estudiante a "hacer filosofía". Pero no creo que tengamos en cuenta la sugerencia de partir de un hecho particular, concreto y contextualizado, de una situación histórica bien definida. Creo que estas recomendaciones no siempre las tenemos. Creo que el problema no es cuando lo llevamos a la práctica sino en el planteo mismo que hacemos de los contenidos.

Saludos

Ana

En estos ejemplos se aprecia cómo se van articulando las intervenciones entre sí, en función del tratamiento del contenido propuesto. La primera intervención (ejemplo 6), es la que ofrece las primeras ideas para trabajar en las intervenciones siguientes. En esta intervención de María se señalan algunos aspectos destacados del texto a trabajar. En estas frases rescata lo que para ella resulta de interés. En la intervención que le sigue (ejemplo 7), Juana recupera las palabras de María las 
analiza y las afirma, sosteniendo que no es una tarea fácil pero que es necesario desarrollarla. Finalmente. en la última intervención de esta articulación (ejemplo 8), Ana recupera las otras dos intervenciones y vuelve a afirmar los aspectos que resultan interesantes del texto para ambas: las cuestiones metodológicas al enseñar filosofía. Luego avanza un paso más al cuestionar la idea planteada por María sobre la problemática que se presenta al pasar dichas recomendaciones a la práctica. Desde este punto de cuestionamiento Ana señala que la cuestión no pasa por la práctica sino por el planteo de los contenidos.

En estas tres intervenciones podemos observar cómo los participantes intercambian sus ideas en función del contenido propuesto a través de diferentes caminos analizando, afirmando, cuestionando. La articulación de estas intervenciones están estrechamente ligadas entre sí, una da lugar a las otras. En la secuencia realizada cada nueva intervención se enlaza con la anterior. Si seguimos analizando la secuencia de intervenciones en este foro se puede observar la misma tendencia. Cada participación recupera elementos de las anteriores y se enlaza directamente a ellas, por uno o varios elementos, afirmados, analizados, cuestionados, etc.

Por otra parte, también se identificaron articulaciones entre la intervención de un participante (tutor o alumnos) que trae a consideración otros textos, autores o ideas que no son los contenidos de referencia planteados por los tutores y profesores (pero que están en relación con los tópicos abordados) y las intervenciones del resto de los compañeros que analizan, cuestionan y retoman estos textos incorporándolos en sus intervenciones.

\section{Ejemplo 9}

Re: ¿Por qué estudiar y enseñar Sociología?

De Angel - miércoles, 8 de abril de 2009, 17:35

Hola, compañeros

José Ortega y Gasset, en su "Orígenes y Epílogos de la Filosofía" afirma que quien vive el mundo y en el mundo de forma natural es un "verdadero vitalicio duermevela" que no supera su estado natural o su estado de animal instintivo. Me parece que esto, atendiendo a la propuesta de la sociología, se refiere al estado del hombre que vive en el mundo sin darse cuenta de su "yo y sus circunstancias", es decir de su responsabilidad de construirse a sí mismo como un ser-en-en-el-mundo en relación con los demás. Pero para que el ser humano sea consciente de esa responsabilidad necesita de las herramientas necesarias u orientaciones externas que la sociedad le debe proveer, 
sobre todo, en sus primeras peregrinaciones en el mundo. Y es aquí la importancia de la sociología, pues ella le orientará a comprender las circunstancias de su mundo social que le condicionan.

\section{Ejemplo 10}

Re: ¿Por qué estudiar y enseñar Sociología?

de Vanesa - jueves, 9 de abril de 2009, 03:31

\section{i Amigos Tan Especiales!}

Estoy muy atenta leyendo cada participación y opinión. Agradezco por las bienvenidas en este grupo. El debate está relevante sobre el estudio y la enseñanza en el social, con base en la lectura del texto de la Profesora. Sobre texto que trae Ángel me parece muy interesante analizar este planteo del "yo y sus circunstancias" el yo en el mundo, su ser en el mundo. Desde la sociología es algo que no podemos dejar de considerar. ella...

Seguimos esperando, la participación de todos es valiosa, por eso contamos con

Que Jesucristo con su infinito amor te bendigas a cada nuevo día.

Con cariño, Vanesa

\section{Ejemplo 11}

Re: ¿Por qué estudiar y enseñar Sociología?

de Mirta - jueves, 9 de abril de 2009, 19:26

Interesante el texto de Ortega y Gasset, me pareció importante lo que destacó Vanesa pero a mí lo que más me llamó la atención es esta cuestión de las herramientas como orientaciones externas que le provee la sociedad al hombre para su desarrollo como sujeto social. Interesante no?, y una gran responsabilidad también.

Cariños

Mirta

En los tres ejemplos señalados se puede observar la articulación de las intervenciones a partir de las palabras de los participantes anteriores en la secuencia del foro. En este caso, lo que produce la articulación de las tres intervenciones es el 
texto de Ortega y Gasset traído a consideración por el primer alumno. A partir de su presentación (ejemplo 9), las siguientes dos participantes realizan indicaciones de ello. En el ejemplo 10, Vanesa recupera algunos detalles del texto aportado por Ángel, y en el ejemplo 11, Mirta recupera las palabras de Vanesa y a su vez señala otros elementos que le resultan significativos.

Una vez más, podemos observar que la articulación no se da por la primera intervención realizada por el tutor, sino por la cadena de intervenciones realiza con anterioridad. Cada intervención se articula con la directamente anterior en la cadena de participaciones realizadas en el foro.

Finalmente, se identificaron articulaciones entre las reflexiones personales que aporta un participante en relación con el tema en discusión, y otros participantes que retoman estas reflexiones para seguir enriqueciendo la discusión. En los siguientes ejemplos se observa cómo la discusión se articula en función de las reflexiones anteriores. Esta vez el contenido de las discusiones ya no es únicamente el contenido trabajado, sino que se suman las propias reflexiones de los participantes que se van enlazando cuidadosamente.

\section{Ejemplo 12}

Re: Tercera semana - Actividad final

De María - jueves, 18 de diciembre de 2008, 14:03

Hola todos y Prof. Siguiendo la reflexión en el foro, considero fundamental, buscar y encontrar la clave del pensamiento filosófico del autor. "Confrontar al filósofo y mirar su pensamiento con relación a los puntos o nodos de su filosofía”. Ayudar al joven a recorrer las calles escondidas, a lograr la salida del laberinto, enseñarle a no quedarse prisionero, sino más bien transformarse en residencia del sujeto, que así puede analizar el significado o significados, frecuentemente ocultos. Por tanto, para llevar a la práctica esta recomendación, el docente es el primero quién debe poseer esa competencia y así podrá guiar al estudiante hacia la comprensión de las ideas claves del pensamiento filosófico propuesto.

El diálogo socrático, es uno de los caminos a recorrer, es la manera adecuada para el desarrollo de la reflexión crítica, en busca de la verdad.

Atentos saludos. María 


\section{Ejemplo 13}

Re: Tercera semana - Actividad final

De Marta - jueves, 18 de diciembre de 2008, 14:39

Siguiendo la reflexión de María, rescato la expresión que dice... "es el docente de filosofía el guía capaz de ayudar al joven a recorrer las calles escondidas".. en la realidad no suele ser tanto así, puesto que en la mayoría de los casos las clases de filosofía suelen ser confusas y aburridas, sin sentido para los alumnos y creo que el desafío para el docente en primer lugar está el considerar la relación de los aspectos teóricos de las materias con sus aplicaciones prácticas en la sociedad, nuestros alumnos comprenderán a los grandes filósofos si comprenden la situación problemática contemporánea y los problemas concretos urgentes, que ellos deben tratar de resolver en sus contextos. y aquí me surge una interrogante ¿̇en qué medida favorecemos en nuestras clases el desarrollo de la capacidad de reflexión crítica, de autonomía y de libertad?

\section{Ejemplo 14}

Re: Tercera semana - Actividad final

De Mirta - jueves, 18 de diciembre de 2008, 18:59

El pensamiento crítico y la autonomía en nuestros alumnos podrán desarrollarse en la medida que se favorezca la reflexión crítica, el proceso de identificación personal y la libertad, se tiene que recuperar la tendencia natural de niños y jóvenes a preguntarse por sí mismos y por el mundo que los rodea, con una actitud de asombro y de cuestionamiento. Discutir en grupos, argumentar y refutar sus ideas, son las posibilidades para promover el aprendizaje autónomo de los alumnos, para que puedan desarrollar todas sus potencialidades como persona, tanto en su dimensión individual como social, adquirir una conciencia crítica es fundamental hoy en día para superar el conformismo, para tomar una postura activa de modo a no perder el derrotero de la existencia y para descifrar los lenguajes que se ocultan detrás de la realidad.

En los ejemplos presentados se pueden analizar cómo las reflexiones de los participantes son la materia prima para el enlace y la articulación de las siguientes intervenciones. En el ejemplo 12, María retoma el contenido del texto trabajado y luego aporta una metáfora que ayuda a su reflexión sobre el tema. En la intervención siguiente (ejemplo 13), Marta recupera una frase de María de su metáfora y la articula son su propia reflexión sobre el tema, planteando hacia el final de la intervención una nueva pregunta "¿ien qué medida favorecemos en nuestras clases el desarrollo de la capacidad de reflexión crítica, de autonomía y de libertad?” Esta pregunta final 
es la que origina la tercera intervención (ejemplo 14) en la cual Mirta da respuesta desde su propio pensamiento sobre el tema.

De esta manera podemos observar una vez más que las intervenciones se enlazan entre si y se articulan en función de elementos manifiestos en las intervenciones directamente anteriores, en este caso producto de las reflexiones personales de los implicados en los debates.

\section{CONCLUSIONES}

A partir del análisis realizado, podemos concluir que nuestra primera intuición acerca de la importancia de la primera intervención para el resto de la interacción comunicativa, que comentamos al inicio de este trabajo, debe ser modificada. Teniendo en cuenta lo analizado hasta el momento, podemos suponer que la primera intervención no sería la guía más relevante de las intervenciones siguientes. sino que las participaciones se articularían entre sí en función de la renovación de los tópicos que se trabajan y los modos de abordarlos. De este modo, vemos que habría una relación más estrecha entre las intervenciones que se suceden seguidamente en una secuencia. Dicha articulación podría ser más frecuente y evidente que la articulación que puede observarse entre la primera intervención y las siguientes, como en primera instancia habíamos intuido.

Este tipo de articulación, a la que llamamos "articulación local", nos permitió realizar el análisis de las intervenciones en las dos dimensiones presentadas, accional y conceptual, y así identificar las particularidades de cada nivel de análisis.

Si bien la caracterización propuesta es aún provisoria, y debe ser verificada en un corpus aún más amplio que el propuesto, consideramos que los próximos pasos en el estudio del fenómeno permitirá proponer estrategias didácticas para optimizar el desempeño de los tutores y los estudiantes en foros y chats de formación. Esto incidiría decisivamente en la facilitación de los procesos de co-construcción del conocimiento en ambientes virtuales de aprendizaje, así como en la optimización de las comunidades de práctica.

\section{NOTAS}

1 Suele considerarse que las actividades se organizan en géneros comunicativos. El género comunicativo comprende significados verbales (y no verbales) que son rutinizados para resolver una tarea. Por ejemplo, un artículo de opinión o un debate. 
2 El modo, o método, específico de resolver (o tratar de resolver) un problema o tarea es denominado estrategia comunicativa. En algunos casos, las estrategias se atribuyen a individuos, mientras que en otros son claramente colectivas.

3 Este master es gestionado por una red institucional latinoamericana y europea a partir de un proyecto ALFA de la UE, con sede administrativa en la Universidad Ca' Foscari di Venecia, y sede operativa en Argentina en el Centro de Investigaciones en Antropología Filosófica y Cultural (CIAFIC), dependiente del Consejo Nacional de Investigaciones Científicas y Técnicas (CONICET).

4 En lingüística y análisis del discurso, este fenómeno se ha estudiado con el nombre de pares adyacentes (e.g. Sacks, Schegloff y Jefferson, 1974; Mey 1993).

$5 \quad$ Siguiendo a Brown y Levinson (1987), consideramos que cada persona tiene una cara social o imagen pública sobre sí, entendida en términos de deseos complementarios:

- La imagen negativa es el deseo de que no nos impongan lo que tenemos que hacer.

- La imagen positiva es el deseo de que los demás aprecien lo que nosotros apreciamos.

Si bien a toda persona le interesa cuidar su imagen, muchos de los actos que realiza son actos que amenazan esa imagen. Algunos amenazan la imagen negativa o positiva del oyente y otros, la imagen positiva o negativa del hablante.

\section{REFERENCIAS BIBLIOGRÁFICAS}

Austin, J. (1962). Cómo hacer cosas con palabras. Madrid: Paidós.

Banzato, M. (2002). Il tutoring in rete. En: Banzato, M. Apprendere in rete. Modelli e strumenti per l e-learning (263-328). Torino: UTET.

Baldry, A.; Thibault, P. J. (2006). Multimodal Transcription and Text Analysis. Londres: Equinox.

Baquero, R. (1997). Vygotsky y el aprendizaje escolar. Buenos Aires: Aique.

Brown G.; Yule, G. (1993). Análisis del discurso. Madrid: Visor.

Bruner, J. (1990). Actos de significado. Madrid: Alianza-Psicología.

Bruner, J. (1997). La educación puerta de la cultura. Madrid: Visor.

Burbules, N. (2000). El diálogo en la enseñanza. Buenos Aires: Amorrortu.

Constantino, G. D. (2002a). Presenza vitale contro presenza virtuale: studio contrastivo dei modi strategico-discorsivi della interazione didattica. En: Banzato,
M. (Ed.) Apprendere in rete. Torino: UTET.

Constantino, G. D. (Comp.) (2002b). Investigación Cualitativa \& Análisis del Discurso en Educación. Catamarca: Universitaria.

Constantino, G. D. (2007). Modalidades comunicativo-discursivas de participación en comunidades virtuales de aprendizaje: una propuesta para la evaluación formativa. Revista Aled, $\mathrm{n}^{\mathrm{0}} 3$, (7-15).

Davis, B. H.; Brewer, J. P. (1997). Electronic Discourse. Linguistic Individuals in Virtual Space. Nueva York: SUNY.

Edwards, D.; Mercer, N. (1988). El conocimiento compartido. El desarrollo de la comprensión en el aula. Barcelona: Paidós.

Eggen, P. D.; Kauchak, D. P. (2001). Estrategias docentes. México: Fondo de cultura económica. 
Fetzer, A. (2002). Communicative Intentions in Context. En: Fetzer, A.; Meierkord, C. (Eds.). Rethinking sequentiality: linguistics meets conversational interaction, cap.3, (37-69). Amsterdam: Benjamins.

Freire, P. (2000). Pedagogía del oprimido. España: Siglo XXI

Garrison, D. R.; Anderson, T. (2005). El elearning en el siglo XXI: Investigación y Práctica. Barcelona: Octaedro.

Gros Salvat, B.; Silva, J. (2006). El problema delanálisisdelas discusionesasincrónicas en el aprendizaje colaborativo mediado. RED: Revista de Educación a Distancia, $\mathrm{N}^{\circ} .16,(1-16)$.

Guba. E. G.; Lincoln, Y. S. (1998). Competing Paradigms in Qualitative research. En: Denzin, N. K.; Lincoln, Y. S. (Eds.). The Landscape of Qualitative Research (195220). Thousand Oak, CA: Sage.

Gunawardena, C.; otros (1997). Analysis of a global online debate and the development o fan interaction análisis model for examining social construction of knowledge in computer conferencing. Journal of Educational Computing Research, $\mathrm{N}^{\circ} 17$, (397-431).

Joyce, B.; Weil, M.; Calhoun, E. (2002). Modelos de enseñanza. Barcelona: Gedisa.

Linell, P. (1998). Approaching dialogue. Amsterdam: Benjamins.

Marková, I.; Linell, P. (1996). Coding elementary contributions to dialogue: Individual acts versus dialogical interactions. Journal for the Theory of Social Behaviour, $\mathrm{n}^{\circ}$ 26, (353-373).

Mercer, N. (1997). La construcción guiada del conocimiento. El habla de profesores y alumnos. Barcelona: Paidós.

Mercer, N. (2000). Words \& Minds. How we use language to think together. Londres: Routledge.

Mey, J. L. (1993). Pragmatics. An introduction. Oxford: Blackwell.
Pardo, M. L. (1994). La gestación del texto: la emisión líder. Tesis doctoral, Buenos Aires: Universidad de Buenos Aires.

Perkins, D. (1997). La escuela inteligente. Del adiestramiento de la memoria a la educación de la mente. Barcelona: Gedisa.

Reigeluth, C. M. (1999). Diseño de la Instrucción. Teorías y modelos. Un nuevo paradigma de la teoría de la instrucción. Tomo II, Aula XXI. Argentina: Santillana.

Reigeluth, C. M. (2009). InstructionalDesign Theories and Models. Vol 3. Nueva York: Routledge.

Reiss, N. (1985). Speech act taxonomy as a tool for ethnographic description. Amsterdam: John Benjamins Publishing Company.

Salomón, G. (Comp.). (1993). Cogniciones distribuidas. Consideraciones psicológicas y educativas. Buenos Aires: Amorrortu.

Salomón, G.; Nevo, B. (2002). Peace education: The concept, principles, and practices around the world. Mahwah, NJ: LEA.

Sánchez-Upegui, A. (2009). Nuevos modos de interacción educativa: análisis lingüístico de un foro virtual. Educación y Educadores, vol. 12, $\mathrm{n}^{\mathrm{O}}$ 2, (29-46).

Sbisá, M. (1992). Speech acts, effects and responses. En: Searle, J. R.; et al (Eds.). (On) Searle on conversation, 9, (101113). Amsterdam Philadelphia: Johns Benjamins Publishing Company.

Searle, J. (1969). Speech acts. An essay in the philosophy of language. New York: Cambridge University Press.

Searle, J. (1975). Actos de habla indirectos. Teorema VII/1, (23-53).

Searle, J. (1979). Expression and meaning. Cambridge: CUP.

Silva, J. (2006). Formación docente en un espacio virtual de aprendizaje: una experiencia concreta en el contexto chileno. Revista Electrónica Teoría de 
la Educación y Cultura en la Sociedad de la Información, $\mathrm{n}^{0}$ 7, (1). [en línea] Disponible en: http://www3.usal. es/ teoriaeducacion/rev numero $07 /$ n7 art silva.htm (consulta 2009, 22 de octubre).
Vasilachis de Gialdino, I. (Coord.) (2007). Estrategias de una investigación cualitativa. Buenos Aires: Editorial Gedisa.

Wenger, E. (1998). Comunidades de práctica. Aprendizaje, significado e identidad. Barcelona: Paidós Ibérica.

\section{PERFIL ACADÉMICO Y PROFESIONAL DE LAS AUTORAS}

Lourdes Morán. Licenciada en Ciencias de la Educación por la Universidad de Buenos Aires, Facultad de Filosofía y Letras. Actualmente se encuentra finalizando las tesis de doctorado en ciencias de la educación y de maestría en Tecnología Educativa en ambas actividades por la misma casa de estudios. Se desempeña como becaria de posgrado tipo II del Consejo Nacional de Investigaciones Científicas y Técnicas en el Centro de Investigaciones en Antropología Filosófica y Cultural. Desde hace unos años se dedica a la profundización en temas vinculados a la didáctica online, en el campo de la articulación entre tecnología y educación en ámbitos universitarios y pre-universitarios. Centro de Investigaciones en Antropología Filosófica y Cultural, Consejo Nacional de Investigaciones Científicas y Técnicas (CIAFIC-CONICET).

E-mail: moran.lourdes1@gmail.com

Guadalupe Álvarez. Doctora en Letras por la Universidad Nacional de Cuyo (Mendoza). Actualmente se desempeña como investigadora asistente del Consejo Nacional de Investigaciones Científicas y Técnicas en el Centro de Investigaciones en Antropología Filosófica y Cultural, y también como investigadora docente en el Instituto del Desarrollo Humano de la Universidad Nacional de General Sarmiento. Desde hace unos años se dedica al estudio del discurso didáctico, presencial y online. Además, está interesada en la didáctica de la lengua en ámbitos preuniversitarios. Por otra parte, la autora ha publicado capítulos de libros y artículos en revistas con referato reconocidas a nivel nacional o internacional.

E-mail: gggalvarez@gmail.com; galvarez@ungs.edu.ar

\section{DIRECCIÓN DE LAS AUTORAS:}

Instituto del Desarrollo Humano

Universidad Nacional de General Sarmiento (IDHUNGS)

Juan María Gutiérrez 1150 (1613)

Los Polvorines, Buenos Aires, Argentina. 
Fecha de recepción del artículo: 21/03/11

Fecha de aceptación del artículo: 30/05/11

\section{Como citar este artículo:}

Morán, L.; Alvarez, G. (2012). Análisis textual-discursivo y pedagógico-discursivo de la interacción comunicativa en foros formativos online: sobre la articulación local de las intervenciones. RIED. Revista Iberoamericana de Educación a Distancia, volumen 15, $\mathrm{n}^{0}$ 1, pp. 51-77. 